Sabrina S. Burgener, Mathias Baumann, Paola Basilico, Eileen Remold-O’Donnell, Ivo P. Touw and Charaf Benarafa*

\title{
Myeloid conditional deletion and transgenic models reveal a threshold for the neutrophil survival factor Serpinb1
}

DOI 10.1515/hsz-2016-0132

Received February 2, 2016; accepted April 20, 2016; previously published online April 22, 2016

\begin{abstract}
Serpinb1 is an inhibitor of neutrophil granule serine proteases cathepsin G, proteinase-3 and elastase. One of its core physiological functions is to protect neutrophils from granule protease-mediated cell death. Mice lacking Serpinb1a $\left(\mathrm{Sbla}^{-/}\right)$, its mouse ortholog, have reduced bone marrow neutrophil numbers due to cell death mediated by cathepsin $\mathrm{G}$ and the mice show increased susceptibility to lung infections. Here, we show that conditional deletion of Serpinb1a using the Lyz2-cre and Cebpa-cre knock-in mice effectively leads to recombination-mediated deletion in neutrophils but protein-null neutrophils were only obtained using the latter recombinase-expressing strain. Absence of Serpinb1a protein in neutrophils caused neutropenia and increased granule permeabilization-induced cell death. We then generated transgenic mice expressing human Serpinb1 in neutrophils under the human MRP8 (S100A8) promoter. Serpinb1a expression levels in founder lines correlated positively with increased neutrophil survival when crossed with $S b 1 a^{-/}$mice, which had their defective neutrophil phenotype rescued in the higher expressing transgenic line. Using new conditional and transgenic mouse models, our study demonstrates the presence of a relatively low Serpinb1a protein threshold in neutrophils that is required for sustained survival.
\end{abstract}

*Corresponding author: Charaf Benarafa, Theodor Kocher Institute, University of Bern, Freiestrasse 1, CH-3012 Bern, Switzerland, e-mail: charaf.benarafa@tki.unibe.ch. http://orcid.org/0000-00022049-7769

Sabrina S. Burgener, Mathias Baumann and Paola Basilico: Theodor Kocher Institute, University of Bern, Freiestrasse 1, CH-3012 Bern, Switzerland; and Graduate School for Cellular and Biomedical Sciences, University of Bern, CH-3012 Bern, Switzerland

Eileen Remold-O’Donnell: Program in Cellular and Molecular Medicine and Division of Hematology/Oncology, Boston Children's Hospital, Boston, MA 02115, USA; and Department of Pediatrics, Harvard Medical School, Boston, MA 02115, USA

Ivo P. Touw: Department of Hematology, Erasmus MC Cancer Institute, 3015 GE, Rotterdam, The Netherlands
These models will also be helpful in delineating recently described functions of Serpinb1 in metabolism and cancer.

Keywords: cell death; cre; serine protease; serpin.

\section{Introduction}

Neutrophils (PMNs) are granulocytes with central functions in inflammatory disease and in innate immunity against microbes (Kruger et al., 2015). Because isolated PMNs are short-lived, they are poorly amenable to in vitro manipulation such as RNA interference and transfection. The use of genetic engineering in mice has thus been helpful in delineating molecular pathways in PMN homeostasis and functions. With this approach, we and others have shown that PMN survival in vivo depends in part on inhibition of granule serine proteases by Serpinb1a (Benarafa et al., 2011; Baumann et al., 2013; Loison et al., 2014). Human SERPINB1 and its mouse homolog Serpinb1a are intracellular inhibitors of neutrophil serine proteases cathepsin G, proteinase-3 and elastase (Cooley et al., 2001; Benarafa et al., 2002). Serpinb1a-deficient mice ( $\left.\mathrm{Sb}^{-\mathrm{a}^{-/}}\right)$ present a profound reduction in PMN survival, leading to a neutropenia in the bone marrow (BM) in steady state conditions as well as in the periphery during infection (Benarafa et al., 2007, 2011). Serpinb1a is expressed broadly in all leukocytes including hematopoietic stem cells (HSCs) but also at high levels in many organs such as lungs, liver, pancreas and prostate (Benarafa et al., 2002). There is an emerging literature correlating variations of Serpinb1 expression with progression of various types of cancers and inflammatory diseases (Ashida et al., 2004; Popova et al., 2006; Yasumatsu et al., 2006; Tseng et al., 2009; Naito et al., 2010; Cui et al., 2014; Zhao et al., 2014; Huasong et al., 2015; Sheng et al., 2015; El Ouaamari et al., 2016). Therefore, additional models to investigate specific functions of this serpin in vivo are needed. Here, we developed and validated tools to conditionally delete Serpinb1a using cre-loxP technology in myeloid cells and, 


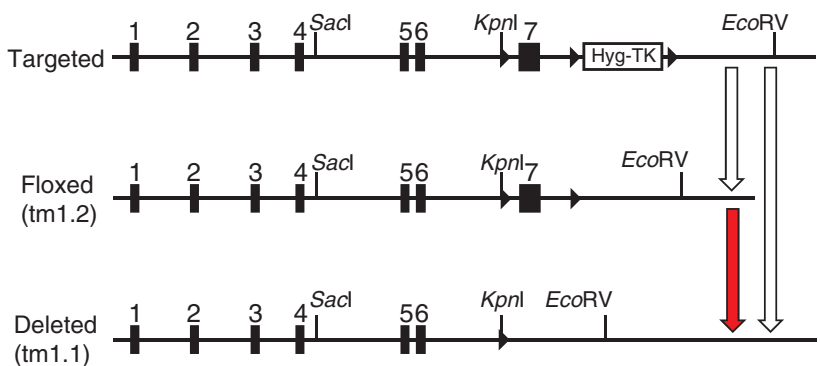

Figure 1: Generation of Serpinb1 $a^{\mathrm{F} / \mathrm{F}}$ mice.

Shown at the top is the targeted Serpinb1a locus with 3-loxP sites (black triangles) in ES cells. Cre recombinase in ES cells (indicated by white arrows) generated two new alleles: either a floxed

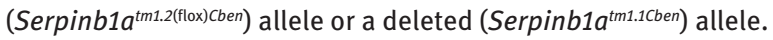
In vivo recombination (red arrow) of a floxed allele in Serpinb1 $1 a^{\mathrm{F} / \mathrm{F}}$ $\left(S b 1 a^{F / F}\right)$ mice is expected to generate a deleted allele in cell lineages expressing cre. Deletion of exon 7 , which encodes the reactive center loop of the serpin, was previously shown to be an effective null allele (Benarafa et al., 2007).

inversely, we generated a transgenic mouse expressing human Serpinb1 (hSerpinb1) in PMNs that rescues the BM neutropenia of $S_{b 1 a^{-/} \text {mice. }}$

\section{Results}

\section{Lyz2-driven cre recombination of Serpinb1a floxed allele in neutrophils}

We have previously targeted Serpinb1a in embryonic stem (ES) cells using a 3-loxP strategy (Benarafa, 2011). Transient transfection of an ES cell clone with a cre expressing plasmid allowed removal of the selection cassette and the generation of ES cell clones with a constitutive deleted allele as well as clones with a conditional allele (Figure 1). The characterization of constitutive $S b 1 a^{-/}$mice derived from ES cells carrying a deleted allele was described previously (Benarafa et al., 2007, 2011). To investigate the phenotype of mice lacking Serpinb1a principally in myeloid cells, conditional $S b 1 a^{\mathrm{F} / \mathrm{F}}$ mice were interbred with the commonly used knock-in mice expressing the cre recombinase from the endogenous Lyz2 (also known as LysM) promoter (Lyz2 $\left.2^{\text {cre/cre }}\right)$ (Clausen et al., 1999). PCR analysis of genomic DNA of sorted BM leukocyte subsets confirmed almost complete cre-mediated recombination of the Serpinb1a locus in PMNs, partial recombination in myelocytes and, as expected, no recombination in B cells (Figure 2A). However, absolute numbers and percentage of PMNs in

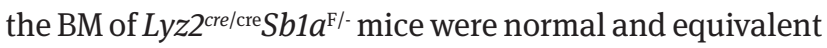
to those of $\mathrm{Sbla}^{+/+}, \mathrm{Sb}_{1} \mathrm{a}^{+/-}$and $\mathrm{Lyz2}^{+++} \mathrm{Sbla}^{\mathrm{F} /}$ mice (Figure 2B), whereas $\mathrm{Sb}_{1} \mathrm{a}^{-}$mice had reduced PMN numbers as we
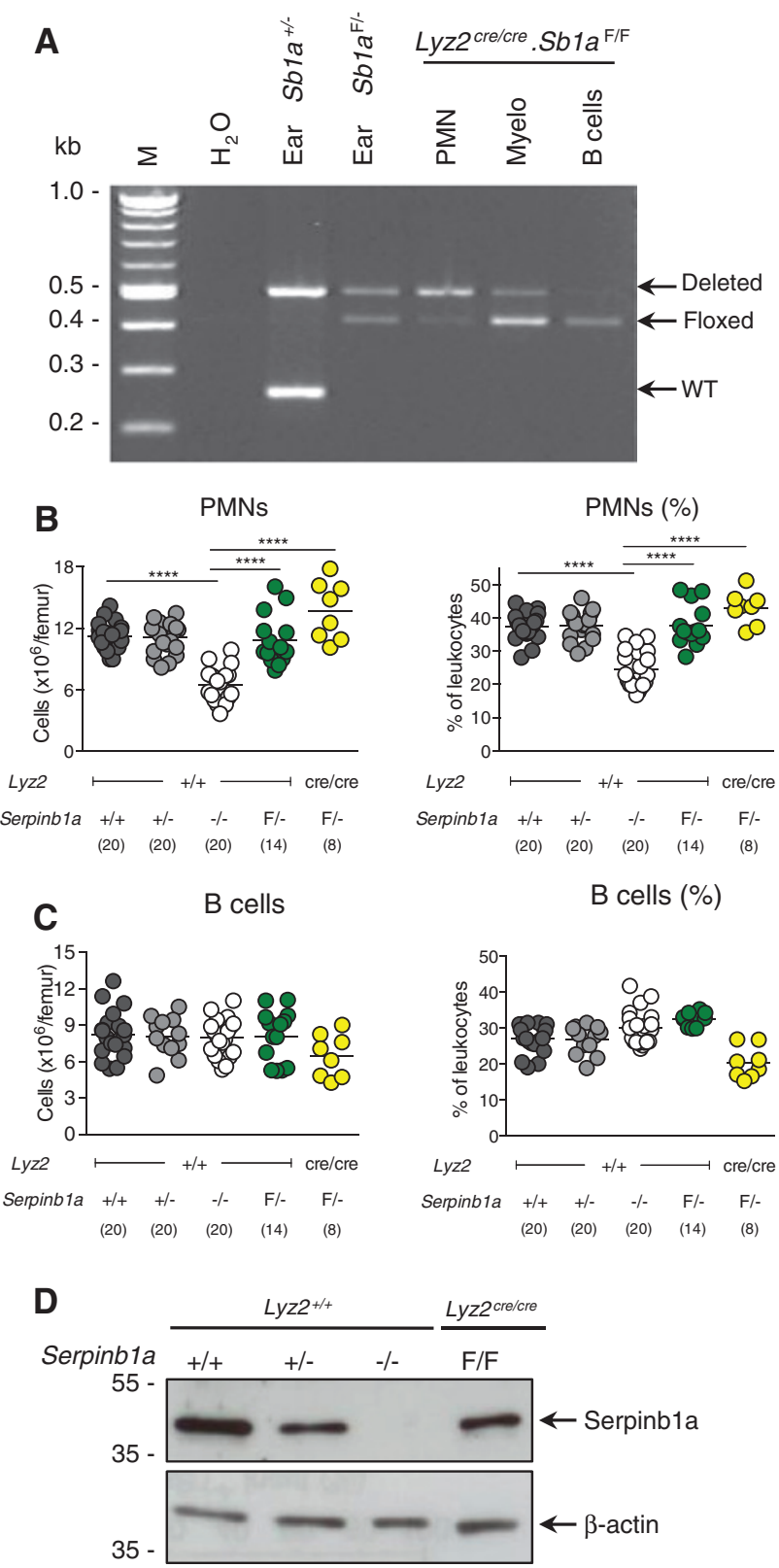

Figure 2: $L y z 2^{\text {cre/cre }}$ deletion of Serpinb1 $a^{F / F}$.

(A) PCR analysis of genomic DNA isolated from flow cytometry sorted $\mathrm{BM}$ cells isolated from $L y z 2^{\text {cre/cre }}$ Serpinb1 $a^{\mathrm{F} / \mathrm{F}}$ mice. Arrows indicate expected size of PCR products for Sb1a alleles (deleted 500bp; floxed 410bp; WT 250bp) as indicated by ear biopsy standard samples. Total number and percentage of BM PMN (B) and B cells (C). BM and blood cell numbers and percentages were analyzed by

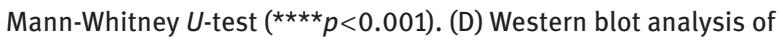
Serpinb1a and $\beta$-actin of flow-sorted BM PMNs.

previously reported (Benarafa et al., 2011; Baumann et al., 2013). As expected, B cell numbers were not significantly different between all genotypes (Figure 2C). Western blot analysis of sorted BM cells revealed that Serpinbla protein was still detectable in PMN lysates of $L y z 2^{\text {cre/cre }} S b 1 a^{\mathrm{F} / \mathrm{F}}$ mice at levels comparable to those of $S b 1 a^{+/-}$mice (Figure 2D). 
These findings demonstrate that $L y z 2^{\text {cre }}$-driven deletion of the Serpinb1a locus does not produce PMNs defective in Serpinb1a protein despite efficient DNA recombination in PMNs.

\section{Cebpa-driven cre recombination of Serpinb1a floxed allele in neutrophils}

To evaluate an alternative model, $S b 1 a^{\mathrm{F} / \mathrm{F}}$ mice were intercrossed with mice expressing the cre recombinase from endogenous CCAAT/enhancer binding protein $\alpha(\mathrm{C} / \mathrm{EBP} \alpha)$ promoter, which drives expression at an earlier developmental stage in myelopoiesis than Lyz2 (Wölfler et al., 2010). Efficient deletion of the floxed allele was observed in PMNs, myelocytes and monocytes but not in B cells (Figure 3A). Western blot analysis also confirmed absence of Serpinb1a protein in sorted PMN lysates of $\mathrm{Cebpa}^{+/ \mathrm{cre}}$ $S b 1 a^{\mathrm{F} /}$ mice (Figure 3B). Accordingly, myeloid cell-specific

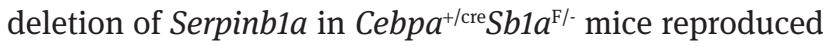
the phenotype of $S b 1 a^{\%}$ mice characterized by reduced absolute numbers and significantly lower percentage of PMNs in the BM (Figure 4A). As in Sb1 ${ }^{-\%}$ mice, other cell subsets in the BM were not altered in $C e b p a^{+/ \text {cre }} S b 1 a^{\mathrm{F} /}$ mice (Figure 3B; Supplementary Figure S1A,B). Numbers of PMNs and other leukocyte subsets in blood as well as other blood parameters were not altered (Figure 4C; Supplementary Figure S1C; Table 1), which is consistent with the phenotype of $\mathrm{Sb}_{1 \mathrm{a}^{-/}}$mice.

PMNs are highly susceptible to granule permeabilization-induced cell death caused by L-leucyl-L-leucine methyl ester (LLME). LLME treatment of PMNs of $\mathrm{Cebpa}^{+/ \text {cre }}$ $S b 1 a^{\mathrm{F} /}$ mice showed reduced survival similar to those of control Cebpa ${ }^{+/ \text {cre Sbla }}{ }^{-1}$ littermates and of $\mathrm{Sbla}^{-1}$ mice (Figure 4D). As shown previously for Sb1a ${ }^{-1}$ PMNs (Baumann et al., 2013), caspase inhibition with Q-VD-OPh had no protective effect on cell death of $\mathrm{Cebpa}^{+/ \mathrm{cre}} \mathrm{Sbla}^{\mathrm{F} / \mathrm{C}}$ PMNs. Taken together, these data demonstrate that myeloid-specific deletion of Serpinb1a largely replicates the PMN phenotype of constitutive $S b 1 a^{-1}$ mice.

\section{Transgenic rescue of $\mathrm{Sb} \mathrm{a}^{-/}$neutrophils with human SERPINB1}

Human SERPINB1 cDNA was cloned downstream of the human S100A8 (MRP8) promoter and was injected in the pronucleus of $\mathrm{C} 57 \mathrm{BL} / 6 \mathrm{~J}$ oocytes. Founder transgenic mice were identified by PCR analysis (Figure 5A). Three of the male founders were crossed with $\mathrm{Sb} \mathrm{a}^{-1}$ mice. Western blot analysis of PMN lysates revealed that the progeny of founder
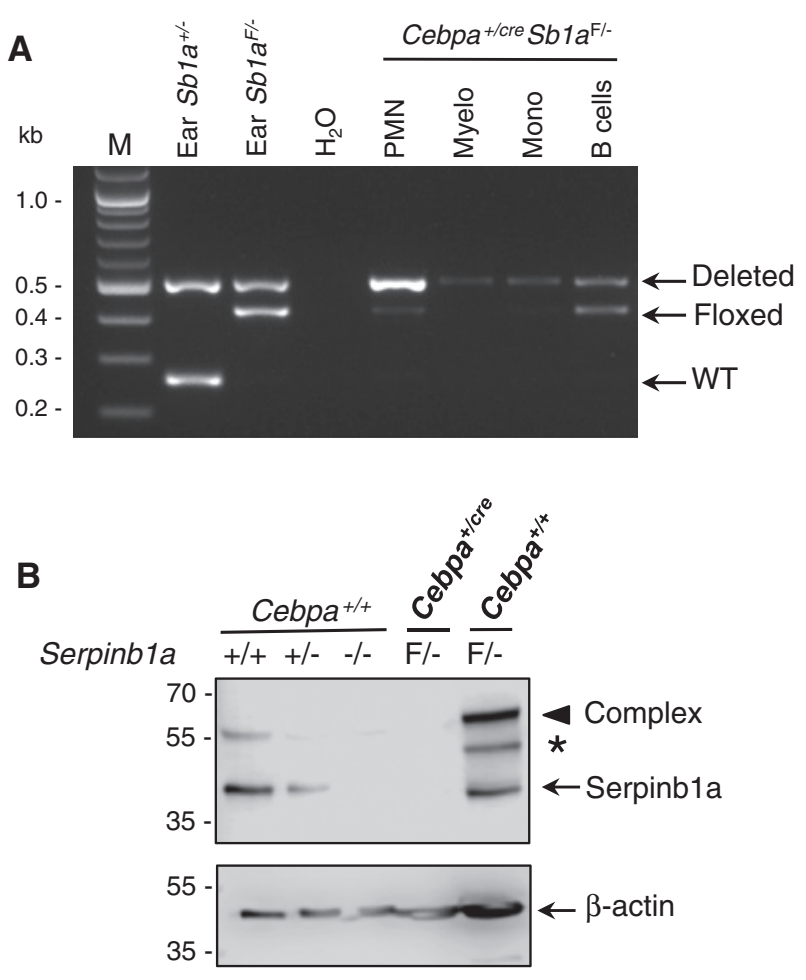

Figure 3: $\mathrm{Cebpa}^{+/ \text {cre }}$ deletion of Serpinb1a.

(A) Genotyping PCR analysis of genomic DNA isolated from flow

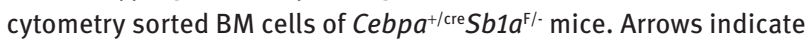
expected size of PCR products for Serpinb1a alleles (deleted 500 bp; floxed 410 bp; WT 250 bp) as indicated by ear biopsy standard samples. (B) Western blot analysis for Serpinb1a and $\beta$-actin of flow-sorted BM PMNs. Serpinb1a-protease complex and partly degraded complex are indicated by an arrowhead and an asterisk, respectively.

3 (Tg3) typically expressed more Serpinb1 protein than the progeny of Tg2 and that Serpinb1 expression was not detectable in the progeny of Tg4 (Figure 5B). Percentage of PMNs in the BM of the different Tg lines correlated with the expression levels of each line (Figure 5C). Correspondingly, protection from LLME-induced granule permeabilization and cell death correlated with Serpinb1 expression levels in the different Tg lines. Specifically, survival of high expressors Tg3 PMNs was rescued to levels of WT PMNs. Reduced survival was observed in PMNs of the Tg2 and Tg4 lines and lowest survival was expectedly observed for Sb1 $a^{-1}$ PMNs (Figure 5D). Therefore, these data demonstrate that Serpinb1 functions as a rheostat in protecting PMNs from granule protease-mediated death in vivo and in vitro.

\section{Discussion}

We report that PMNs lacking Serpinb1a protein were successfully generated in mice expressing the cre 
A Bone marrow PMNS

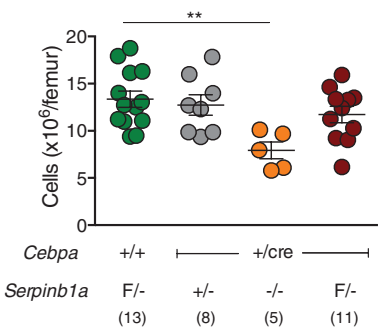

B Bone marrow monocytes

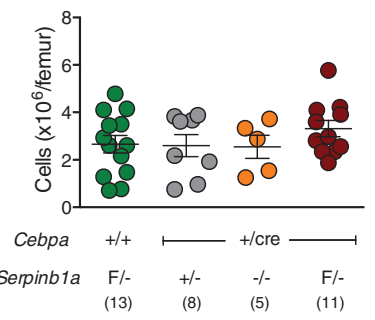

C

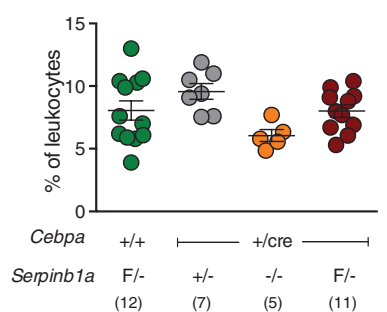

D

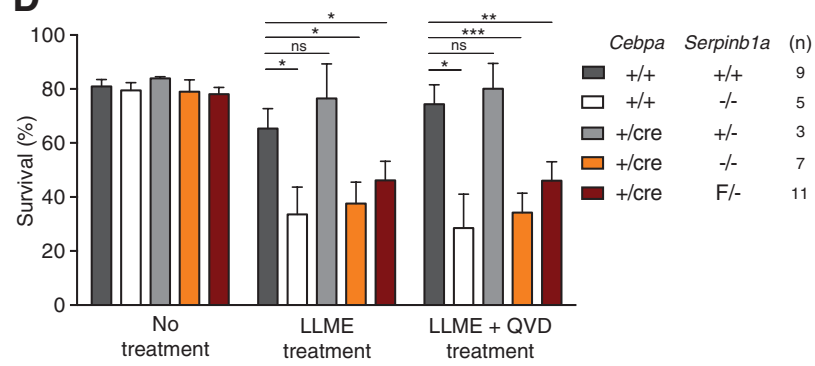

Figure 4: Deletion of Serpinb1a in myeloid cells is sufficient to reduce PMN survival.

Total number and percentage of BM PMNs (A), BM monocytes (B). Percentage of blood PMNs and monocytes (C). BM cell numbers and percentages were analyzed by Mann-Whitney $U$-test $\left({ }^{\star \star} p<0.01\right)$. (D) Survival of PMNs treated with LLME $(100 \mu \mathrm{M})$ in the presence or absence of the caspase inhibitor Q-VD-OPh $(50 \mu \mathrm{M})$. Viability was assessed using Annexin V-FITC and 7- AAD staining of Ly-6G cells and analyzed by Student's $t$-test relative to wild-type PMNs ( $\left.{ }^{\star} p<0.05 ;{ }^{* \star} p<0.01 ;{ }^{* \star *} p<0.001\right)$.

recombinase driven by the Cebpa promoter but not by the $L y z 2$ promoter. Accordingly, PMNs of Cebpa ${ }^{+/ \text {cre }} S b 1 a^{\mathrm{F} /}$ mice, but not PMNs of $L y z 2^{\text {cre/cre }} S b 1 a^{\mathrm{F} /}$ mice, recapitulated the phenotype of $S b 1 a^{-1}$ PMNs with reduced survival in vitro and in vivo. We found that the floxed Serpinb1a gene was essentially recombined in mature PMNs in both models. Failure to generate Serpinbla protein-null PMNs despite efficient genomic recombination in $L y z 2^{\text {cre/cre }} S b 1 a^{\mathrm{F} /-}$ mice is likely due to a combination of factors. First, the floxed Serpinb1a locus was not fully recombined during early stages of granulopoiesis. Second, low levels of Serpinb1a protein may suffice to support PMN survival. Since Serpinb1a transcription is active in HSCs and peaks early in granulopoiesis at the promyelocyte/myelocytes stages (Benarafa et al., 2011), Serpinb1a protein is already accumulating in the cell before the floxed gene is recombined. Thus, sufficient Serpinb1a protein levels may be sustained throughout PMNs' short life-span and maintain normal PMN levels in the BM of $L y z 2^{\text {cre/cre }} S b 1 a^{\mathrm{F} /-}$ mice.

PMNs develop in the BM from hematopoietic stem cells via bipotent granulocyte/macrophage progenitors (GMPs), which can develop into monocyte or PMN lineages. Cebpa is expressed at high levels at the GMP stage, whereas Lyz2 expression peaks later and independently in both granulocyte and monocyte lineages. Cremediated recombination using these two cre knock-in models has been widely and successfully used to delete genes in the myeloid compartment. Yet, as we have seen, the timing of cre expression as well as the lineage may be important in achieving effective gene recombination in early stages of granulopoiesis. Recombination efficiency at different stages of hematopoiesis also differs depending on the targeted locus and therefore making the right choice of a cre deleter strain can be difficult and will always require experimental confirmation. Our study highlights that it remains crucial that gene deletion and protein levels are effectively measured particularly when recombination appears to have no effect on the studied phenotype as shown by the absence of BM neutropenia in $L y z 2^{\text {cre/cre }} S b 1 a^{\mathrm{F} /-}$ mice. Far from a theoretical question, in studies where protein levels were not verified and showing no phenotype in mice with deleted myeloid cells (Rupec et al., 2005; Kirkland et al., 2012), incomplete protein deletion may have led to overlooking the contribution of the studied proteins in myeloid cells.

We showed here that the extent of the rescue of PMN survival by transgenic expression of human SERPINB1 in $S b 1 a^{-/}$mice was dependent on transgene expression levels. Evaluating the threshold levels of Serpinb1 necessary for cytoprotection is challenging because Serpinb1-protease complexes are processed rapidly into post-complex cleavage forms when proteases are in excess (Cooley et al., 2011). Furthermore, the antibody used in Western blot analysis may react differently with endogenous mouse and transgenic human Serpinb1 as well as with the different complexes. In the $S b 1 a^{-/} \mathrm{Tg} 2$ progeny, the hSerpinb1 transgene is relatively weakly expressed and only detectable in 
Table 1: Hematological analysis of whole blood.

\begin{tabular}{|c|c|c|c|c|c|c|}
\hline Genotype & WBC $\left(\times 10^{6}\right.$ cells $\left./ \mathrm{ml}\right)$ & $\mathrm{RBC}\left(\times 10^{9}\right.$ cells $\left./ \mathrm{ml}\right)$ & PLT $\left(\times 10^{6}\right.$ cells $\left./ \mathrm{ml}\right)$ & Hemoglobin (g/dl) & Hematocrit (\%) & $\mathrm{n}$ \\
\hline$S b 1 a^{+/+}$ & $6.84 \pm 3.4$ & $9.57 \pm 0.4$ & $1295 \pm 248$ & $15.8 \pm 0.7$ & $52.7 \pm 2.6$ & 8 \\
\hline$S b 1 a^{+/-}$ & $6.41 \pm 1.6$ & $9.38 \pm 0.7$ & $1309 \pm 160$ & $15.6 \pm 1.2$ & $48.2 \pm 5.4$ & 7 \\
\hline$S b 1 a^{\%}$ & $6.13 \pm 2.9$ & $9.57 \pm 0.6$ & $1656 \pm 129$ & $16.3 \pm 0.4$ & $51.2 \pm 3.0$ & 4 \\
\hline$S b 1 a^{F /-}$ & $6.06 \pm 2.6$ & $9.66 \pm 0.7$ & $1479 \pm 182$ & $16.2 \pm 1.0$ & $50.4 \pm 4.3$ & 13 \\
\hline $\mathrm{Cepba}^{+/ \mathrm{cre}} \mathrm{Sb} 1 \mathrm{a}^{+/-}$ & $6.47 \pm 2.4$ & $9.55 \pm 0.7$ & $1361 \pm 258$ & $15.9 \pm 1.2$ & $49.9 \pm 5.1$ & 8 \\
\hline $\mathrm{Cepba}^{+/ \mathrm{cre}} \mathrm{S} b 1 a^{-/}$ & $5.46 \pm 0.8$ & $9.49 \pm 0.7$ & $1532 \pm 187$ & $15.7 \pm 1.0$ & $50.3 \pm 2.9$ & 5 \\
\hline $\mathrm{Cepba}^{+/ \mathrm{cre}} \mathrm{S} b 1 a^{\mathrm{F} /-}$ & $5.69 \pm 1.2$ & $9.80 \pm 0.6$ & $1348 \pm 142$ & $16.1 \pm 0.9$ & $51.0 \pm 4.4$ & 9 \\
\hline
\end{tabular}

A

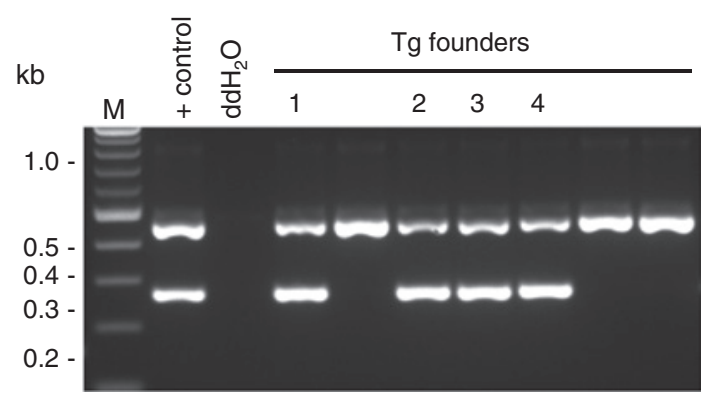

B

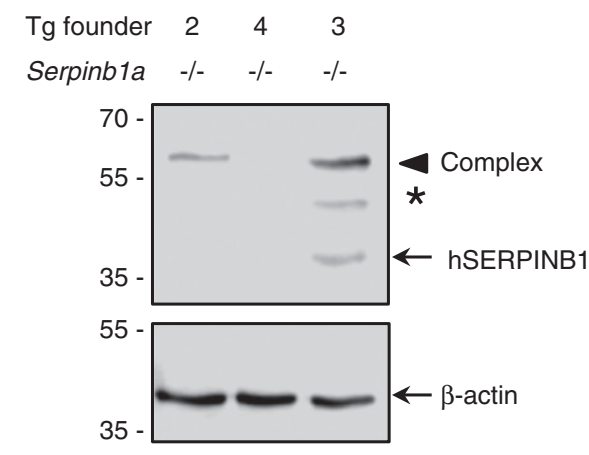

C

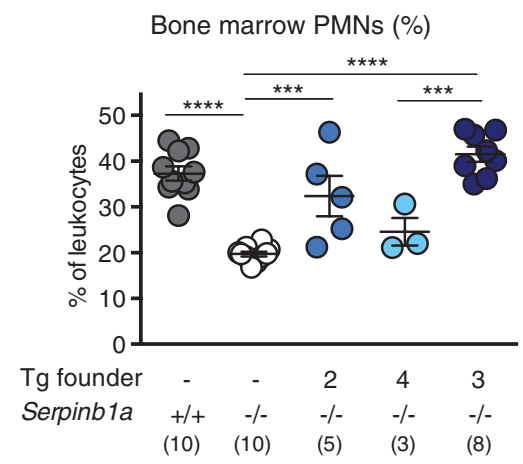

D

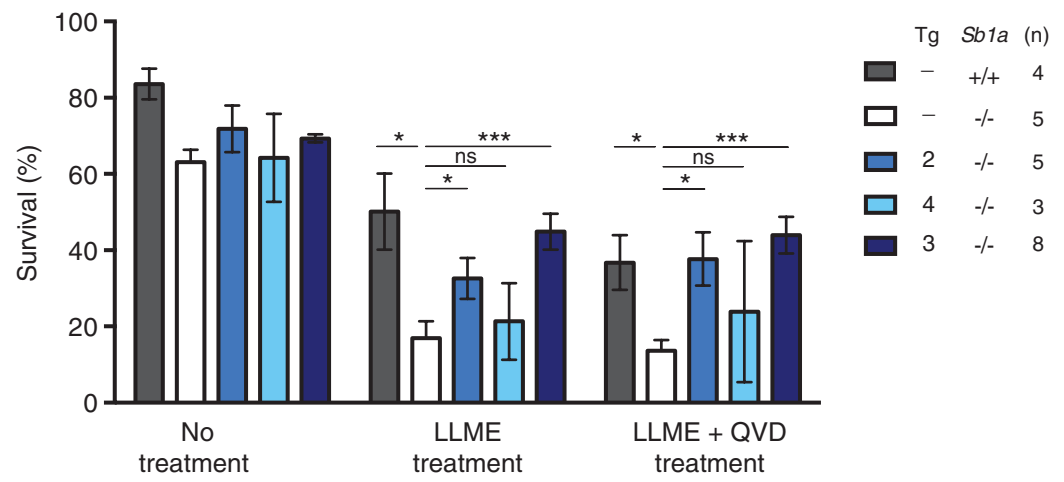

Figure 5: Rescue of Serpinb1a $\%$ PMN survival by transgenic expression of human SERPINB1.

(A) PCR analysis of genomic DNA of transgenic founders using a single primer pair that recognizes both the human SERPINB1 transgene CDNA (266 bp) and mouse Serpinb1a gene (460 bp, which includes an intronic sequence). (B) Western blot analysis for SERPINB1 and $\beta$-actin of flow-sorted BM PMNs from offsprings of Tg founders 2, 3 and 4. hSerpinb1-protease complex and partly degraded complex are indicated by an arrowhead and an asterisk, respectively. (C) Percentage of PMN numbers in the BM of Sb1 $a^{-/} \cdot \operatorname{Tg} 2, S_{1} 1 a^{-/} \operatorname{Tg} 3 \mathrm{and}^{\mathrm{S}} \mathrm{Sb} 1 a^{-/} \mathrm{Tg} 4 \mathrm{mice}$ $\left.{ }^{\star \star} p<0.01\right)$. (D) Survival of PMNs treated with LLME $(100 \mu \mathrm{M})$ in the presence or absence of the caspase inhibitor Q-VD-OPh (50 $\left.\mu \mathrm{m}\right)$. Viability was assessed using Annexin V-FITC and 7- AAD staining of Ly- $6 \mathrm{G}^{+}$cells and analyzed by Student's $t$-test relative to $S b 1 a^{-/ \cdot}$ PMNs $\left({ }^{*} p<0.05\right.$; $\star \star \star p<0.001)$. 
complex with proteases. With all the caveats in mind for such a comparison, it suggests that the $\mathrm{Tg} 2$ line expresses substantially lower levels than heterozygous $\mathrm{Sbla}^{+/-}$mice, where active mouse Serpinb1a can be detected. Yet, in the $S b 1 a^{-/} \mathrm{Tg} 2$ mice, we observed a partial rescue of the survival phenotype in vivo and of LLME-induced death in vitro. In the $S b 1 a^{-/} \mathrm{Tg} 4$ progeny, the transgene was not detectable by Western blot analysis, suggesting very low or no expression and, accordingly, PMN numbers in the BM were as low as in Sb1a ${ }^{-/}$mice. In the higher expressing transgenic line (Tg3), hSERPINB1 transgene was found in active and complex forms and PMN survival was fully rescued in vitro and in vivo. This new transgenic model will allow us to evaluate the function of the ubiquitously expressed Serpinb1a in non-hematopoietic tissues in the absence of the PMN survival defect in various disease models.

More targeted deletions in tissue or cell subsets could then follow using $S b 1 a^{\mathrm{F} /}$ mice crossed with other cre-expressing mouse lines. Of note, reporter analysis of $\mathrm{Cebpa}^{+/ \text {cre }}$ mice showed recombination in the liver and lung airway epithelium (Wölfler et al., 2010). It is thus likely that the $S b 1 a^{\mathrm{F} / \cdot}$ locus was recombined in some nonhematopoietic cells of $\mathrm{Cebpa}^{+/ \mathrm{cre}} \mathrm{Sb} 1 \mathrm{a}^{\mathrm{F} /}$ mice. We previously demonstrated that, in BM chimera, deletion of Sb1a in the hematopoietic compartment was necessary and sufficient to reproduce the phenotype of $S b 1 a^{--}$mice (Baumann et al., 2013). Therefore, effective deletion of Serpinb1a in myeloid cells is undoubtedly the cause of BM neutropenia in $\mathrm{Cebpa}^{+/ \mathrm{cre} S} \mathrm{Sb}^{\mathrm{F}} \mathrm{F}^{\mathrm{F}-}$ mice. However, careful scrutiny of Sb1a expression in various cell types and time points will be required when using $\mathrm{Cebpa}^{+/ \mathrm{cre}} \mathrm{Sb} 1 a^{\mathrm{F} /}$ mice in systemic

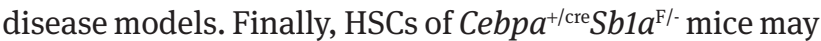
be most useful to reconstitute the immune system of irradiated mice to generate myeloid-specific deficiency.

Transgenic mice expressing cre under the hMRP-8 promoter (S100A8) and knock-in models such as neutrophil elastase knock-in mice (Elane tm1(cre)Roes) $^{\text {or Ly6G }}$ knock-in (Catchup) mice ( ${ }^{2} 6 g^{\text {tm2621(Cre-ddTomato)Arte })}$ are additional models for PMN-specific gene deletion in PMNs (Tkalcevic et al., 2000; Passegué et al., 2004; Hasenberg et al., 2015). These models have reduced targeting of the monocytic lineage than Lyz2 and Cebpa cre knock-in mice used here. Whether Serpinb1a protein-null PMNs can be obtained using these models remains to be tested but is unlikely given the persistence of the protein in PMNs

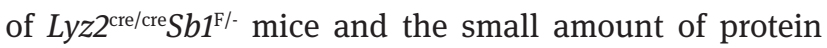
rescue needed for mitigating the $S b 1 a^{-1}$ phenotype in transgenic mice. For example, deletion of the Fcgr4 gene was complete and specific for PMNs at the genomic level in Catchup mice. While the mice showed a specific phenotype due to gene deletion, FcRIV expression on the PMN surface measured by flow cytometry was only reduced by 50\% (Hasenberg et al., 2015). Experimental approaches using cre-mediated recombination in PMNs thus remain challenging in choosing the right cre-expressing model(s) and in the interpretation of the data. In addition to time and resources to generate the mice, it requires specific attention to the target gene expression pattern, protein stability and functional protein threshold to choose the right cre-expressing model(s) and to draw appropriate conclusions.

In summary, we showed that the survival of PMNs depends on a threshold level of Serpinb1 below which serine protease activity is not controlled, leading to cell death. These findings are consistent with the mode of action of Serpinb1 as a stoichiometric inhibitor of neutrophil proteases. Our study further supports the notion that intracellular serpins such as clade B serpins in vertebrates and serpins of Caenorhabditis elegans have a fundamental cytoprotective function (Bird, 1999; Zhang et al., 2006; Luke et al., 2007; Tan et al., 2013; Bird et al., 2014). We have previously demonstrated that BM neutropenia of $\mathrm{Sb} \mathrm{a}^{-/}$mice is dependent on cathepsin $\mathrm{G}$ in vivo (Baumann et al., 2013). In addition, Serpinb1 prevents spontaneous PMN apoptosis by inhibiting proteinase-3-mediated cleavage and activation of caspase-3 (Loison et al., 2014). PMNs are exquisitely sensitive to death after granule leakage induced by LLME treatment and cathepsin G is required for this caspase-independent cell death pathway that is critically regulated by Serpinb1 (Baumann et al., 2013). Similarly, Serpinb9 protects cytotoxic lymphocytes against granzyme B-mediated death following granule leakage induced by LLME treatment or by T cell activation (Bird et al., 2014). Serpinb6a is another intracellular serpin inhibitor of cathepsin $\mathrm{G}$ that is expressed in PMNs and may contribute to the protease shield against granule permeability-induced cell death. Ongoing studies using multiple targeting of the large locus of clade B serpin locus on mouse chromosome 13 will determine the relative contributions of Serpinb1a, Serpinb6a, Serpinb9 and additional understudied serpin paralogs in cellular homeostasis. The models described here will provide important tools for these studies.

\section{Materials and methods}

\section{Ethics statement}

All animal studies were approved by the Cantonal Veterinary Office of the canton of Bern and conducted in accordance with the Swiss federal legislation on animal welfare. 


\section{Mouse models for conditional deletion of Serpinb1a}

Serpinb1a ${ }^{--}$(Serpinb1 $\left.{ }^{\text {tm.1.1cben }}\right)$ mice were generated in 129S6/SvEvTac (129S6) background (Benarafa et al., 2007) and backcrossed in C57BL/6J background (Benarafa et al., 2011). The latter were used in this study. Serpinb1a $a^{F / F}$ (Serpinb1 $a^{\text {tm1.2(flox)Cben }}$ ) were generated in parallel with Sb1 ${ }^{\digamma}$ mice. Briefly, 129S6/W4 ES cells (Taconic) were targeted by homologous recombination with a linearized plasmid described previously (Benarafa, 2011). Homologous recombinant clones with 3-loxP sites were transiently transfected with Cre recombinase to excise the floxed CMV-HYG/TK positive/negative selection cassette. Cells were further selected with gancyclovir to eliminate the clones where the deletion of the selection cassette did not occur. While most clones tested had recombined the first and third loxP sites to generate the deleted allele (serpinB1 $a^{\text {tm.1.1cben }}$ ), we also found clones that recombined the second and third loxP sites, leaving an allele $\left(\operatorname{serpinB1} a^{\text {tm1.2(flox)Cben }}\right.$ ) with a floxed exon 7 (Figure 1). ES cells (clone 2F7-F5) were injected into blastocysts at the transgenic core facility of the Brigham and Women Hospital (Boston). Sb1 $a^{+/ F}$ heterozygous mice were generated from chimeric mice. During backcrossing into C57BL/6J for 10 generations, $S b 1 a^{+/ F}$ breeders were also selected based on the polymorphic PCR markers D13Mit117 and D13Mit16 to reduce the portion of chromosome 13 belonging to the $129 \mathrm{~S} 6$ strain to $<3-4 \mathrm{cM}$ on each side of the Serpinb1a locus. Cre recombinase knock-in mice Lyz $^{\text {cre/cre }}$ mice (B6.129P2-Lyz2 $2^{\text {tml(cre)Ifo) }}$ (Clausen et al., 1999) were obtained from the Jackson Laboratories at backcross generation N6 and were further backcrossed to N10 with C57BL6/J mice before intercrossing with $S b 1 a^{F / F}$ mice to generate the desired genotypes. Cre recombinase knock-in mice Cebpa ${ }^{+/ \text {cre }}$ mice (Cebpa $\left.{ }^{\text {tmI(cre)Touw }}\right)$ were described previously (Wölfler et al., 2010).

\section{Generation of human SERPINB1 transgenic mice}

A $3.8 \mathrm{~kb}$ fragment upstream of the $S 100 A 8$ start codon was amplified by PCR from a human BAC clone obtained from imaGenes (I.M.A.G.E. clone number RPCIB75301168Q) and directionally cloned using NheI and SalI restriction digest upstream of human SERPINB1 cDNA flanked downstream by the SV40 small T intron and poly-adenylation signal. The final DNA construct was verified by sequencing and the plasmid backbone was excised by restriction digest with ClaI and NotI. The transgene band was separated by agarose electrophoresis and gel purified. The transgene was microinjected in C57BL/6J oocytes at the Cryoconservation and Transgenic unit of the Theodor Kocher Institute, University of Bern. Four male and four female mice out of 21 total mice born were positive for the transgene by PCR. Three of the four transgene positive founder males transmitted the transgene to their progeny and were then crossed with $\mathrm{Sbla}^{-/}$mice. The transgene was always maintained as hemizygous. The progeny of each founder were handled as independent transgenic lines named $\operatorname{Tgx}$ (full nomenclature B6J-Tg(S100A8-SERPINB1)xCben), where ' $\mathrm{x}$ ' represents the founder number.

\section{Flow cytometry and cell sorting}

Leukocyte counts and hematopoietic lineage differential analysis of BM was performed in mice aged 6-8-weeks old. BM cells were harvested from femurs by flushing with PBS supplemented with $1 \%$ FCS, red blood cells were lyzed with ammonium chloride for $10 \mathrm{~min}$, further washed in PBS and counted in a Neubauer chamber. Isolated BM cells were stained with fluorescently labeled antibodies (Biolegend and BD Biosciences) and analyzed on a FACScalibur flow cytometer (BD Biosciences) as described previously. Briefly, leukocyte subsets percentages were determined within $\mathrm{CD}^{2} 5^{+}$cells as PMNs (CD11b ${ }^{+} \mathrm{Ly}-$ $\left.6 \mathrm{G}^{+}\right)$, monocytes $\left(\mathrm{CD} 115^{+}\right)$, myelocytes $\left(\mathrm{CD} 11 \mathrm{~b}^{+}, \mathrm{CD} 115^{\text {neg }}, \mathrm{Ly}_{6} \mathrm{G}^{\text {neg }}\right.$, $\mathrm{SSC}^{\text {high }}$ ) and B cells (CD19+ or CD45RB220 ${ }^{+}$. Blood was collected by retro-orbital venous puncture using heparinized microcapillary tubes and leukocyte subsets analyzed by flow cytometry as above. Total leukocyte, erythrocyte and platelet counts as well as hemoglobin and hematocrit measurements of whole blood were performed using a Scil Vet ABC hematology analyzer (Horiba Medical, Montpellier, France). Flow cytometry data was analyzed using FlowJo (FlowJo LLC). Flow sorting of PMNs, B cells and myelocytes was performed on single-cell suspensions of BM leukocytes stained with antibodies mentioned above using a FACS Aria II sorter (BD Biosciences) at the flow cytometry core facility of the Department of Clinical Research of the University of Bern.

\section{Western blotting}

Sorted cells were washed and lysed $\left(10^{7} / \mathrm{ml}\right)$ in RIPA buffer with protease inhibitor cocktail (Roche). Lysates were resolved by SDSPAGE under reducing conditions and immunoblotted using rabbit antiserum to human SERPINB1 provided by ERO (Rees et al., 1999). Blots were stripped and restained with anti- $\beta$-actin antibody (Cell Signaling Technology).

\section{Cell death induced by granule permeabilization}

BM cells were cultured in DMEM (4 mm L-Glut, $25 \mathrm{~mm}$ D-Glucose, $1 \mathrm{~mm}$ sodium pyruvate) (Life Technologies) containing $1 \%$ FCS and $1 \%$ penicillin/streptomycin at $1.0 \times 10^{6}$ cells $/ \mathrm{ml}$ in the presence or absence of the pan-caspase inhibitor Q-VD-OPh (SM Biochemicals LLC) or LLME (G-2550; Bachem). Cells were harvested and viability was assessed using annexin V-fluorescein isothiocyanate (FITC) and 7-aminoactinomycin D (7AAD) and measured at the FACScalibur flow cytometer.

\section{Statistical analysis}

Leukocyte subset analysis was performed using Mann-Whitney $U$-test or Student's $t$-test with GraphPad Prism Mac 4.0c software (GraphPad, San Diego, CA, USA). A $p$ Value $<0.05$ was considered statistically significant.

Acknowledgments: We thank Elisabeth Frei and Stephan Hirschi for excellent technical assistance. We acknowledge Albert Witt for pronucleus microinjection, Lina Du for ES cell injection and Bernadette Nyfeler for cell sorting. We thank the ZEMB and DKF animal caretaker teams for dedicated attention and husbandry. This study was supported by grants from the Swiss National Science Foundation 
(127464 and 149790) (CB), the EU/FP7 Marie Curie International Reintegration Grant (CB) grant 249297 and the Bern University Research Foundation (CB). Serpinb1 ${ }^{+/ F}$ mice were generated with support from the National Institutes of Health grant HL66548 (ERO).

\section{References}

Ashida, S., Nakagawa, H., Katagiri, T., Furihata, M., liizumi, M., Anazawa, Y., Tsunoda, T., Takata, R., Kasahara, K., Miki, T., et al. (2004). Molecular features of the transition from prostatic intraepithelial neoplasia (PIN) to prostate cancer: genome-wide gene-expression profiles of prostate cancers and PINs. Cancer Res. 64, 5963-5972.

Baumann, M., Pham, C.T.N., and Benarafa, C. (2013). SerpinB1 is critical for neutrophil survival through cell-autonomous inhibition of cathepsin G. Blood 121, 3900-3907.

Benarafa, C. (2011). The SerpinB1 knockout mouse a model for studying neutrophil protease regulation in homeostasis and inflammation. Methods Enzymol. 499, 135-148.

Benarafa, C., Cooley, J., Zeng, W., Bird, P.I., and Remold-O'Donnell, E. (2002). Characterization of four murine homologs of the human ov-serpin monocyte neutrophil elastase inhibitor MNEI (SERPINB1). J. Biol. Chem. 277, 42028-42033.

Benarafa, C., Priebe, G.P., and Remold-O'Donnell, E. (2007). The neutrophil serine protease inhibitor serpinb1 preserves lung defense functions in Pseudomonas aeruginosa infection. J. Exp. Med. 204, 1901-1909.

Benarafa, C., LeCuyer, T.E., Baumann, M., Stolley, J.M., Cremona, T.P., and Remold-O'Donnell, E. (2011). SerpinB1 protects the mature neutrophil reserve in the bone marrow. J. Leukoc. Biol. 90, 21-29.

Bird, P.I. (1999). Regulation of pro-apoptotic leucocyte granule serine proteinases by intracellular serpins. Immunol. Cell Biol. 77, 47-57.

Bird, C.H., Christensen, M.E., Mangan, M.S.J., Prakash, M.D., Sedelies, K.A., Smyth, M.J., Harper, I., Waterhouse, N.J., and Bird, P.I. (2014). The granzyme B-Serpinb9 axis controls the fate of lymphocytes after lysosomal stress. Cell Death Differ. $21,876-887$.

Clausen, B.E., Burkhardt, C., Reith, W., Renkawitz, R., and Förster, I. (1999). Conditional gene targeting in macrophages and granulocytes using LysMcre mice. Transgenic Res. 8, 265-277.

Cooley, J., Takayama, T.K., Shapiro, S.D., Schechter, N.M., and Remold-O'Donnell, E. (2001). The serpin MNEl inhibits elastase-like and chymotrypsin-like serine proteases through efficient reactions at two active sites. Biochemistry 40 , 15762-15770.

Cooley, J., Sontag, M.K., Accurso, F.J., and Remold-O’Donnell, E. (2011). SerpinB1 in cystic fibrosis airway fluids: quantity, molecular form and mechanism of elastase inhibition. Eur. Respir. J. 37, 1083-1090.

Cui, X., Liu, Y., Wan, C., Lu, C., Cai, J., He, S., Ni, T., Zhu, J., Wei, L., Zhang, Y., et al. (2014). Decreased expression of SERPINB1 correlates with tumor invasion and poor prognosis in hepatocellular carcinoma. J. Mol. Histol. 45, 59-68.

El Ouaamari, A., Dirice, E., Gedeon, N., Hu, J., Zhou, J.-Y., Shirakawa, J., Hou, L., Goodman, J., Karampelias, C., Qiang, G., et al. (2016). SerpinB1 promotes pancreatic $\beta$ cell proliferation. Cell Metab. 23, 194-205.

Hasenberg, A., Hasenberg, M., Männ, L., Neumann, F., Borkenstein, L., Stecher, M., Kraus, A., Engel, D.R., Klingberg, A., Seddigh, P., et al. (2015). Catchup: a mouse model for imaging-based tracking and modulation of neutrophil granulocytes. Nat. Methods 12, 445-452.

Huasong, G., Zongmei, D., Jianfeng, H., Xiaojun, Q., Jun, G., Sun, G., Donglin, W., and Jianhong, Z. (2015). Serine protease inhibitor (SERPIN) B1 suppresses cell migration and invasion in glioma cells. Brain Res. 1600, 59-69.

Kirkland, D., Benson, A., Mirpuri, J., Pifer, R., Hou, B., DeFranco, A.L., and Yarovinsky, F. (2012). B cell-intrinsic MyD88 signaling prevents the lethal dissemination of commensal bacteria during colonic damage. Immunity 36, 228-238.

Kruger, P., Saffarzadeh, M., Weber, A.N.R., Rieber, N., Radsak, M., von Bernuth, H., Benarafa, C., Roos, D., Skokowa, J., and Hartl, D. (2015). Neutrophils: between host defence, immune modulation, and tissue injury. PLoS Pathog. 11, e1004651.

Loison, F., Zhu, H., Karatepe, K., Kasorn, A., Liu, P., Ye, K., Zhou, J., Cao, S., Gong, H., Jenne, D.E., et al. (2014). Proteinase 3-dependent caspase-3 cleavage modulates neutrophil death and inflammation. J. Clin. Invest. 124, 4445-4458.

Luke, C.J., Pak, S.C., Askew, Y.S., Naviglia, T.L., Askew, D.J., Nobar, S.M., Vetica, A.C., Long, O.S., Watkins, S.C., Stolz, D.B., et al. (2007). An intracellular serpin regulates necrosis by inhibiting the induction and sequelae of lysosomal injury. Cell 130, 1108-1119.

Naito, Y., Takagi, T., Okada, H., Omatsu, T., Mizushima, K., Handa, O., Kokura, S., Ichikawa, H., Fujiwake, H., and Yoshikawa, T. (2010). Identification of inflammation-related proteins in a murine colitis model by $2 \mathrm{D}$ fluorescence difference gel electrophoresis and mass spectrometry. J. Gastroenterol. Hepatol. 25(Suppl 1), S144-S148.

Passegué, E., Wagner, E.F., and Weissman, I.L. (2004). JunB deficiency leads to a myeloproliferative disorder arising from hematopoietic stem cells. Cell 119, 431-443.

Popova, E.Y., Claxton, D.F., Lukasova, E., Bird, P.I., and Grigoryev, S.A. (2006). Epigenetic heterochromatin markers distinguish terminally differentiated leukocytes from incompletely differentiated leukemia cells in human blood. Exp. Hematol. 34, 453-462.

Rees, D.D., Rogers, R.A., Cooley, J., Mandle, R.J., Kenney, D.M., and Remold-O’Donnell, E. (1999). Recombinant human monocyte/ neutrophil elastase inhibitor protects rat lungs against injury from cystic fibrosis airway secretions. Am. J. Respir. Cell Mol. Biol. 20, 69-78.

Rupec, R.A., Jundt, F., Rebholz, B., Eckelt, B., Weindl, G., Herzinger, T., Flaig, M.J., Moosmann, S., Plewig, G., Dörken, B., et al. (2005). Stroma-mediated dysregulation of myelopoiesis in mice lacking I $\mathrm{KB} \alpha$. Immunity 22, 479-491.

Sheng, L., Anderson, P.H., Turner, A.G., Pishas, K.I., Dhatrak, D.J., Gill, P.G., Morris, H.A., and Callen, D.F. (2015). Identification of vitamin D3 target genes in human breast cancer tissue. J. Steroid Biochem. Mol. Biol. http://dx.doi.org/10.1016/j. jsbmb.2015.10.012 (in press).

Tan, J., Prakash, M.D., Kaiserman, D., and Bird, P.I. (2013). Absence of SERPINB6A causes sensorineural hearing loss with multiple histopathologies in the mouse inner ear. Am. J. Pathol. 183, 49-59. 
Tkalcevic, J., Novelli, M., Phylactides, M., Iredale, J.P., Segal, A.W., and Roes, J. (2000). Impaired immunity and enhanced resistance to endotoxin in the absence of neutrophil elastase and cathepsin G. Immunity 12, 201-210.

Tseng, M.-Y., Liu, S.-Y., Chen, H.-R., Wu, Y.-J., Chiu, C.-C., Chan, P.-T., Chiang, W.-F., Liu, Y.-C., Lu, C.-Y., Jou, Y.-S., et al. (2009). Serine protease inhibitor (SERPIN) B1 promotes oral cancer cell motility and is over-expressed in invasive oral squamous cell carcinoma. Oral Oncol. 45, 771-776.

Wölfler, A., Danen-van Oorschot, A.A., Haanstra, J.R., Valkhof, M., Bodner, C., Vroegindeweij, E., van Strien, P., Novak, A., Cupedo, T., and Touw, I.P. (2010). Lineage-instructive function of C/EBP $\alpha$ in multipotent hematopoietic cells and early thymic progenitors. Blood 116, 4116-4125.

Yasumatsu, R., Altiok, O., Benarafa, C., Yasumatsu, C., BingolKarakoc, G., Remold-O’Donnell, E., and Cataltepe, S. (2006).
SERPINB1 upregulation is associated with in vivo complex formation with neutrophil elastase and cathepsin $\mathrm{G}$ in a baboon model of bronchopulmonary dysplasia. Am. J. Physiol. Lung Cell Mol. Physiol. 291, L619-L627.

Zhang, M., Park, S.-M., Wang, Y., Shah, R., Liu, N., Murmann, A.E., Wang, C.-R., Peter, M.E., and Ashton-Rickardt, P.G. (2006). Serine protease inhibitor 6 protects cytotoxic T cells from selfinflicted injury by ensuring the integrity of cytotoxic granules. Immunity 24, 451-461.

Zhao, P., Hou, L., Farley, K., Sundrud, M.S., and Remold-O'Donnell, E. (2014). SerpinB1 regulates homeostatic expansion of IL-17 ${ }^{+} \delta$ and CD4 ${ }^{+}$Th17 cells. J. Leukoc. Biol. 95, 521-530.

Supplemental Material: The online version of this article (DOI: 10.1515/hsz-2016-0132) offers supplementary material, available to authorized users. 